\title{
Replica Treatment of the Calogero-Sutherland Model
}

\author{
Dimitry M. Gangardt and Alex Kamenev \\ Department of Physics, Technion, 32000 Haifa, Israel
}

October 31, 2018

\begin{abstract}
Employing Forrester-Ha method of Jack polynomials, we derive an integral identity connecting certain $N$-fold coordinate average of the Calogero-Sutherland model with the $n$-fold replica integral. Subsequent analytical continuation to non-integer $n$ leads to asymptotic expressions for the (static and dynamic) density-density correlation function of the model as well as the Green's function for an arbitrary coupling constant $\lambda$.
\end{abstract}

\section{Introduction}

The model of one-dimensional particles interacting via the inverse square potential, known as the Calogero-Sutherland model (CSM) [1], has attracted a great deal of attention [2, 3, 4, 5]. It has long been realized [1] that for the three special values of the interaction parameter $\lambda=1 / 2,1,2$ the CSM is closely related to the random matrix theory (RMT) [6] of the three Wigner-Dyson ensembles: orthogonal, unitary and symplectic respectively. Recently, using the method of Jack polynomials, Forrester [2] found the correlation functions of the CSM for any integer $\lambda$. The method was latter generalized by Ha [5] to any rational $\lambda=p / q$, where $p$ and $q$ are mutual primes. In the latter case the density-density correlation function of the model may be written as the $(p+q)$-fold integral over momenta of $p$ hole and $q$ particle excitations [4, 0 . This seemingly makes the answer a highly irregular function of the coupling strength $\lambda$.

In this paper we employ the replica method, borrowed from the theory of disordered systems 17] and specifically RMT [8, 9, 10], to treat the CSM at an arbitrary value of $\lambda$. The idea is to write the particle density operator as the $n$-th power of the characteristic polynomial in the limit $n \rightarrow 0$. In the context of the CSM, this appears to be a particularly simple operator which creates at most $n$ holes and no other excitations [5]. As a result, for an integer $n$, the correlation function of the characteristic polynomials may be expressed as an $n$-fold integral over hole momenta for any coupling constant, $\lambda$.

Mathematically, this statement boils down to the powerful asymptotic $(N \rightarrow \infty)$ identity between the $N$-fold coordinate average of the $n$-th power of the characteristic polynomial and the $n$-fold momenta integral. For $\lambda=1$ (unitary RMT) such integral representation has been obtained earlier by Verbaarschot and Zirnbauer [8] by mapping the problem onto the non-linear $\sigma$-model. We derive here the identity valid for any $\lambda$ employing the Forrester-Ha method of Jack polynomials. As of today we are not aware if this identity has a geometrical or field-theoretical derivation apart from $\lambda=1 / 2,1,2$. The simplicity of the final expression suggests the possibility that such a derivation exists. We stress, however, that for any $\lambda$ the identity has clear quantummechanical interpretation. We hope also that it may prove to be useful in the theory of the Coulomb gases.

The calculation requires evaluation of the resulting $n$-fold integral for any integer $n$. We accomplish this goal in the asymptotic regime $x \gg 1$ ( $x$ is the scaled distance) by summing up contributions on $n+1$ saddle points $(n-1$ of these saddle points may be dubbed as "replica non-symmetric"). The subsequent analytic continuation, $n \rightarrow 0$, is analogous to the one employed recently in the RMT context [9, 10. Some mathematical aspects of this procedure are not 
completely clear at the moment 11]. For rational coupling constants $\lambda$ our procedure is in exact agreement with the existing results [5, 12] and allows one to obtain them in a much simpler way. In addition it leads to expressions formally valid for any $\lambda$.

The paper is organized as follows. In section 2 we define the CSM and introduce the replica method. In section 3 we derive the integral identity discussed above. Section 1 is devoted to the calculation of the $n$-fold integral in the large $x$ limit and analytical continuation, $n \rightarrow 0$. Finally, our results and some conclusions are summarized in section 5 . Technical details are presented in three appendices.

\section{CSM and replica}

\subsection{Eigenstates of CSM}

Consider $N$ interacting particles on the unit circle. The CSM [1] is defined by the following Hamiltonian:

$$
H=\sum_{j=1}^{N}\left(z_{j} \partial_{j}\right)^{2}+2 \sum_{j<i} \frac{\lambda(\lambda-1)}{\left|z_{j}-z_{i}\right|^{2}},
$$

where $z_{j}=e^{i \theta_{j}}$ are the coordinates of the $j$-th particle, $\partial_{j}=\partial / \partial z_{j}$ and $\lambda$ is the coupling constant. The first term is the usual kinetic energy, while the second one describes inverse square interactions. The spectrum and the many-body eigenfunctions of the model may be found exactly using the theory of the symmetric functions [13, 14]. For the sake of completeness and to set notations we present here the basic features of this solution (for more details see Appendix A).

The ground state wave-function of the CSM 11 is 15

$$
\langle z \mid 0\rangle=\Psi_{0}(z)=\sqrt{\mathcal{N}}\left[\Delta_{N}(z)\right]^{\lambda},
$$

where

$$
\Delta_{N}(z) \equiv \prod_{j<i}\left(z_{i}-z_{j}\right)
$$

is the $N \times N$ Vandermonde determinant and the normalization constant is given by

$$
\mathcal{N}=\mathcal{N}(\lambda, N)=(2 \pi)^{-2 N} \frac{\Gamma^{N}(1+\lambda)}{\Gamma(1+\lambda N)} .
$$

Excited states are labeled by the quantum numbers (essentially momenta) $(\kappa)=\left(\kappa_{1}, \kappa_{2}, \ldots, \kappa_{N}\right)$, where the non-negative integers $\kappa_{j}$ are ordered as $\kappa_{1} \geq \ldots \geq \kappa_{N} \geq 0$. In the mathematical literature the quantum numbers $(\kappa)$ are called partitions [16] and represented by the Young diagrams, with $\kappa_{j}$ cells in $j$-th row. The example of the Young diagram for the partition $(\kappa)=(7,6,5,5,3,2)$ sometimes written as $\left(7,6,5^{2}, 3,2\right)$ is presented in Fig. 田. In our case number of rows in a Young diagram cannot exceed $N$, whereas number of columns is unlimited. The state $(\kappa)$ has well defined total momentum, whose value is given by the number of cells in the corresponding Young diagram and denoted as $|\kappa| \equiv \sum_{j=1}^{N} \kappa_{j}$. The coordinate representation wave-function of the excited state corresponding to a given partition $(\kappa)$ may be written as

$$
\langle z \mid(\kappa)\rangle=\Psi_{(\kappa)}(z)=\sqrt{\mathcal{N}_{(\kappa)}} J_{(\kappa)}^{1 / \lambda}(z) \times\left[\Delta_{N}(z)\right]^{\lambda},
$$

where the symmetric functions $J_{(\kappa)}^{1 / \lambda}(z)=J_{(\kappa)}^{1 / \lambda}\left(z_{1}, \ldots, z_{N}\right)$ are known as Jack polynomials 13, 14. Definitions and some properties of Jack polynomials along with the normalization constants, $\mathcal{N}_{(\kappa)}$, are presented in Appendices A, B. 

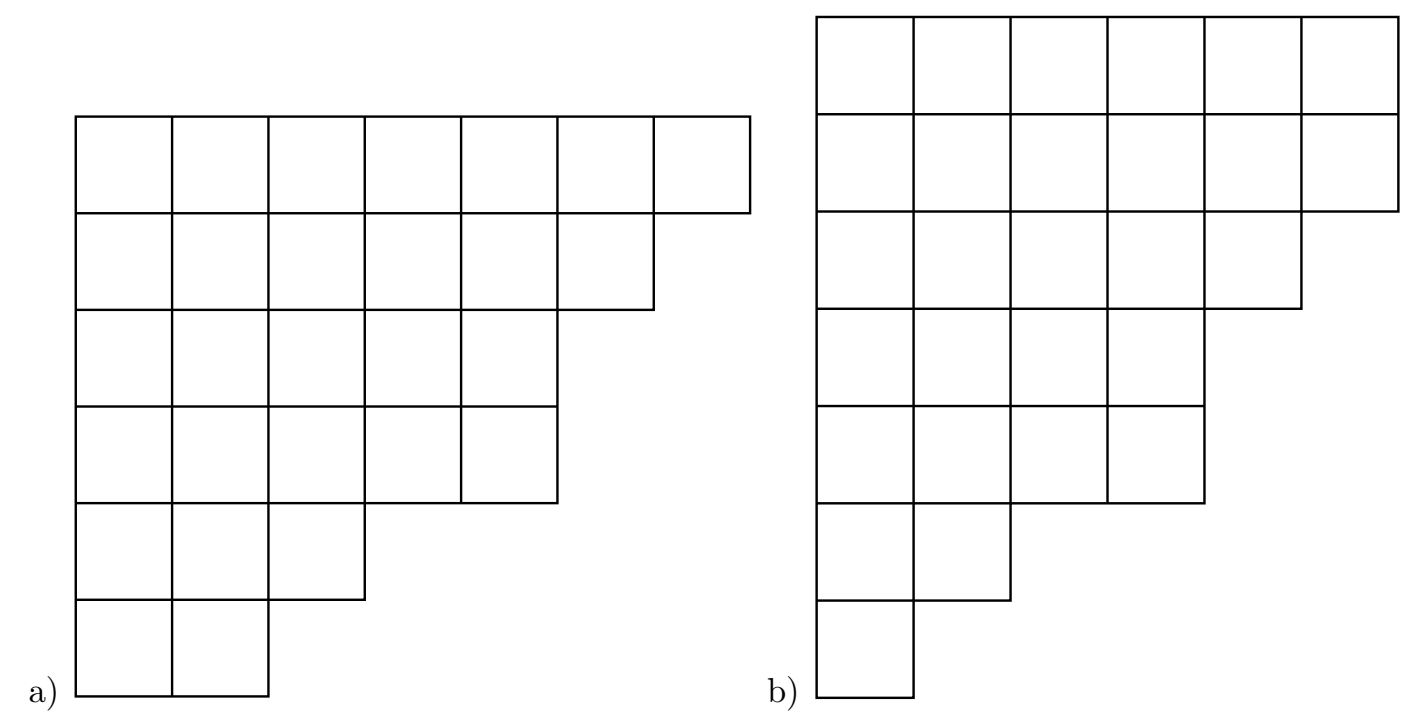

Figure 1: a) Young diagram for partition $(\kappa)=(7,6,5,5,3,2)$ representing an excitation of particles in the Calogero-Sutherland model. b) The Young diagram for the dual partition $(\tilde{\kappa})=(6,6,5,4,4,2,1)$ representing the excitation of holes.

\subsection{Correlation functions}

We focus first on the ground state, static density-density correlation function of the CSM:

$$
R(\theta) \equiv \rho_{0}^{-2}\langle 0|\tilde{\rho}(\theta) \tilde{\rho}(0)| 0\rangle,
$$

where $\tilde{\rho}(\theta)=\rho(\theta)-\rho_{0}$ denotes the fluctuating part of the density operator $\rho(\theta)=\sum_{j} \delta\left(\theta-\theta_{j}\right)$ and $\rho_{0}=N / 2 \pi$ is the mean density. Due to the translational invariance of the model this correlation function depends only on the relative angle between the two points. One may represent the fluctuating part of the density operator as the Fourier series:

$$
\tilde{\rho}(\theta)=\sum_{k \neq 0} \rho_{k} e^{-i k \theta}
$$

where the Fourier coefficients of the density, $\rho_{k}=\bar{\rho}_{-k}$, are given by

$$
2 \pi \rho_{k}=\sum_{j=1}^{N} e^{i k \theta_{j}}=\sum_{j=1}^{N} z_{j}^{k} .
$$

In the theory of symmetric functions the Fourier coefficients $2 \pi \rho_{k}$ are referred to as power sums of variables $z_{j}$. Since the Jack polynomials constitute a complete basis in the ring of symmetric polynomials the power sums $\rho_{k}$ can be decomposed into linear combinations of $J_{(\kappa)}^{1 / \lambda}(z)$, which is the basis of Ha's [5] approach. Here we employ a different method.

Let us define the characteristic polynomial (or vertex) operator as

$$
Z(\theta) \equiv \prod_{j=1}^{N}\left(1-e^{-i\left(\theta-\theta_{j}\right)}\right)=\prod_{j=1}^{N}\left(1-e^{-i \theta} z_{j}\right) .
$$

In the RMT this object is known as the characteristic polynomial of the unitary matrix with eigenvalues $z_{j}$ or spectral determinant. 
Rewriting the characteristic polynomial in the form

$$
Z(\theta)=\exp \left\{\sum_{j=1}^{N} \ln \left(1-e^{-i \theta} z_{j}\right)\right\}=\exp \left\{-2 \pi \sum_{k=1}^{\infty} \frac{\rho_{k} e^{-i k \theta}}{k}\right\},
$$

one notices that the fluctuating part of the density is given by

$$
\tilde{\rho}(\theta)=\frac{1}{\pi} \partial_{\theta} \operatorname{Im} \ln Z(\theta) .
$$

To ensure convergence of the series in Eq. (10) one should assume an infinitesimal negative imaginary part of $\theta: \theta \rightarrow \theta-i 0$. The correlation function Eq. (6) may be expressed therefore through the correlation of logarithms of the spectral determinants:

$$
R(\theta)=-\frac{\rho_{0}^{-2}}{2 \pi^{2}} \partial_{\theta}^{2} \operatorname{Re}\langle 0|\ln \bar{Z}(\theta) \ln Z(0)| 0\rangle .
$$

The replica trick is to write the logarithm as

$$
\ln Z=\lim _{n \rightarrow 0} \frac{1}{n}\left(Z^{n}-1\right) .
$$

As a result one finally obtains for the correlation function

$$
R(\theta)=-\lim _{n \rightarrow 0} \frac{1}{2 \pi^{2} n^{2}} \frac{\partial^{2}}{\partial\left(\rho_{0} \theta\right)^{2}} \operatorname{Re}\left\langle 0\left|\bar{Z}^{n}(\theta) Z^{n}(0)\right| 0\right\rangle,
$$

If one was able to calculate the expectation value on the r.h.s. for any real $n$ the limiting procedure would be straightforward. The non-trivial aspects of the replica method show up if the calculation is possible only for positive 21] integer $n$. As we shall see in the next section this is indeed the case.

\section{Correlations of characteristic polynomials}

In this section we calculate the large $N$ limit of the correlation function of the characteristic polynomials for integer $n$. In the coordinate representation it takes the form of the following $N$-fold integral

$$
\left\langle 0\left|\bar{Z}^{n}(\theta) Z^{n}(0)\right| 0\right\rangle=\mathcal{N} \int_{0}^{2 \pi} d^{N} \theta\left|\Delta_{N}(z)\right|^{2 \lambda}\left[\prod_{j=1}^{N}\left(1-e^{i \theta} \bar{z}_{j}\right)\left(1-z_{j}\right)\right]^{n},
$$

where the normalization constant is given by Eq. (四).

Consider the ground state expectation value of the $s$-th power of the characteristic polynomials, where $s$ is an arbitrary real number. Inserting resolution of unity, one finds

$$
\left\langle 0\left|\bar{Z}^{s}(\theta) Z^{s}(0)\right| 0\right\rangle=\sum_{(\kappa)}\left\langle 0\left|\bar{Z}^{s}(\theta)\right|(\kappa)\right\rangle\left\langle(\kappa)\left|Z^{s}(0)\right| 0\right\rangle=\sum_{(\kappa)}\left|\left\langle(\kappa)\left|Z^{s}(0)\right| 0\right\rangle\right|^{2} e^{i|\kappa| \theta} .
$$

where the sum runs over all possible partitions (with number of rows less than $N$ ). The last equality in Eq. (16) is a trivial consequence of the translational invariance and the fact that the state $|(\kappa)\rangle$ carries momentum $|\kappa|$. Since $Z^{s}(0)$ is a symmetric function of $z$ it may be expanded in the basis of Jack polynomials. Employing the orthogonality relation for Jack polynomials, one obtains the matrix elements $\left\langle(\kappa)\left|Z^{s}(0)\right| 0\right\rangle$. Details of this calculation are given in Appendix B. The result is

$$
\left\langle 0\left|\bar{Z}^{s}(\theta) Z^{s}(0)\right| 0\right\rangle=\sum_{(\kappa)}\left|A_{(\kappa)}(\lambda, N)\right|^{2}\left(\{-s\}_{(\kappa)}^{\lambda}\right)^{2} e^{i|\kappa| \theta},
$$


where $A_{(\kappa)}(\lambda, N)$ is an $s$-independent factor, which is given in Appendix $\mathrm{B}$ and the symbol $\{-s\}_{(\kappa)}^{\lambda}$ is defined as

$$
\{-s\}_{(\kappa)}^{\lambda}=\prod_{j, a \in(\kappa)}(-s-\lambda(j-1)+(a-1)) .
$$

Here the product runs over all cells of the Young diagram for a given partition $(\kappa)$ with $j=1, \ldots, N$ being the vertical coordinate and $a=1,2, \ldots$ the horizontal coordinate of the cell.

At this point the advantage of the replica approach may be appreciated. One notices that for $s=n$ - positive integer, the factor $\{-n\}_{(\kappa)}^{\lambda}$ defined by Eq. (18) is zero for any partition $(\kappa)$ which has more than $n$ columns in its Young diagram. Indeed, for $j=1$ (the upper row) and $a=n+1$ the factor on the r.h.s of Eq. (18) nullifies for any $\lambda$. As a result the set of partitions which contribute to the correlation function, Eq. (17), is greatly reduced. It contains only partitions which have not more than $N$ rows and not more than $n$ columns in their Young diagrams.

The similar drastic reduction in the number of contributing partitions takes place in the Ha's approach [5]. However, in that case it happens only for rational $\lambda=p / q$ and the geometry of the relevant Young diagrams is determined by $p$ and $q$. In the replica approach the fact that $n$ is integer takes care of diagrams selection, whereas $\lambda$ may be arbitrary. In other words, the operator $Z^{n}$ is a particularly simple one: by acting on the ground state it creates not more than $n$ hole-type excitations. As a result, number of partitions (excited states) needed to represent $Z^{n}|0\rangle$ is finite and each partition can be parametrized by the heights of its $n$ columns.

The next step is to take the thermodynamic limit, $N \rightarrow \infty$. To this end we parameterize the relevant partitions by the height of the columns in their Young diagrams. This introduces the dual partition $(\tilde{\kappa})=\left(\tilde{\kappa}_{1}, \tilde{\kappa}_{2}, \ldots, \tilde{\kappa}_{n}\right)$ whose Young diagram is just a transposition of the diagram for the partition $(\kappa)$. Since Young diagram of $(\kappa)$ has no more than $N$ rows and no more than $n$ columns, $0 \leq \tilde{\kappa}_{a} \leq N$ and $a=1,2 \ldots n$. Physically $\tilde{\kappa}_{a}$ describe momenta of $n$ holes; each cannot exceed $N$ since the "Fermi" sea is bound (recall that $\kappa_{j}$ describe unbounded momenta of $N$ particles). In the limit $N \rightarrow \infty$ one may pass from summations over $\tilde{\kappa}_{a}$ in Eq. (17) to integration over $k_{a} \equiv \tilde{\kappa}_{a} / N$, where $0 \leq k_{a} \leq 1$, simultaneously taking the limit in $A_{(\kappa)}(\lambda, N \rightarrow \infty)$. The corresponding procedure is described in details in Appendix C. As a result one finds in the large $N$ limit:

$$
\left\langle 0\left|\bar{Z}^{n}(\theta) Z^{n}(0)\right| 0\right\rangle=N^{\frac{n^{2}}{\lambda}} \mathcal{C}(\lambda, n) \int_{0}^{1} d^{n} k\left|\Delta_{n}(k)\right|^{2 / \lambda}\left[\prod_{a=1}^{n} k_{a}\left(1-k_{a}\right)\right]^{1 / \lambda-1} \exp \left\{i N \theta \sum_{a=1}^{n} k_{a}\right\},
$$

where the normalization constant is

$$
\mathcal{C}(\lambda, n)=\prod_{a=1}^{n} \frac{\Gamma(1+1 / \lambda)}{\Gamma(1+a / \lambda) \Gamma(a / \lambda)} .
$$

Finally, employing Eqs. (15) and (田), one may express the result as the identity of the two integrals

$$
\begin{aligned}
\lim _{N \rightarrow \infty} N^{-\frac{n^{2}}{\lambda}} \mathcal{N}(\lambda, N) & \int_{0}^{2 \pi} d^{N} \theta\left|\Delta_{N}(z)\right|^{2 \lambda}\left[\prod_{j=1}^{N}\left(1-e^{2 \pi i x / N} \bar{z}_{j}\right)\left(1-z_{j}\right)\right]^{n} \\
=\mathcal{C}(\lambda, n) & \int_{0}^{1} d^{n} k\left|\Delta_{n}(k)\right|^{2 / \lambda}\left[\prod_{a=1}^{n} k_{a}\left(1-k_{a}\right)\right]^{1 / \lambda-1} \exp \left\{2 \pi i x \sum_{a=1}^{n} k_{a}\right\}
\end{aligned}
$$

where we have introduced the scaling variable $x \equiv \rho_{0} \theta=N \theta /(2 \pi)$ which measures distance in units of the mean inter-particle separation, to emphasize that the left hand side has a well defined thermodynamic limit.

For $\lambda=1$ (unitary RMT), the identity Eq. (21) was derived by Verbaarschot and Zirnbauer [8], using fermionic replica [21] and mapping the random matrix problem onto the non-linear 
$\sigma$-model on the coset space $U(2 n) / U(n) \times U(n)$. In that formulation parameters $k_{a}$ play the role of the (compact) angular coordinates on the coset. In a similar way one may derive Eq. (21) for $\lambda=1 / 2$ and 2 (orthogonal and symplectic RMT) [17]. It would be extremely interesting to see if there is geometrical field-theoretical interpretation of Eq. (21) for other $\lambda$.

The integral identity Eq. (21) is exact for any $\theta$. In particular, for $x=0$ its right hand side may be evaluated with the help of the Selberg integral [6] leading to

$$
\lim _{N \rightarrow \infty} N^{-\frac{n^{2}}{\lambda}}\left\langle 0\left|\bar{Z}^{n}(0) Z^{n}(0)\right| 0\right\rangle=\prod_{a=1}^{n} \frac{\Gamma(a / \lambda)}{\Gamma((a+n) / \lambda)}:
$$

result obtained previously by Keating 18] for $\lambda=1$. For $x \neq 0$ the integral on the r.h.s. can not be evaluated exactly. In the next section we shall evaluate it in the asymptotic limit $x \gg 1$ employing the stationary phase method as suggested by Forrester [2] and Yurkevich and Lerner [10].

\section{Replica Limit}

For $x \gg 1$ the dominant contribution to the integral on the r.h.s. of Eq. (21) comes from the end points of the interval. We expand the integrand in $l$ variables about 1 and in remaining $n-l$ variables about 0 to leading order. Extending limits of integration to infinity, one obtains for the integral:

$$
\sum_{l=0}^{n}\left(\begin{array}{l}
n \\
l
\end{array}\right) I_{n-l}(-2 \pi i x) I_{l}(2 \pi i x) e^{2 \pi i x l}
$$

where $I_{m}$ are Selberg integrals [6] :

$$
I_{m}(z)=\int_{0}^{\infty} d^{m} k\left|\Delta_{m}(k)\right|^{2 / \lambda} \prod_{a=1}^{m} k_{a}^{1 / \lambda-1} e^{-z k_{a}}=z^{-\frac{m^{2}}{\lambda}} \prod_{a=1}^{m} \frac{\Gamma(1+a / \lambda) \Gamma(a / \lambda)}{\Gamma(1+1 / \lambda)} .
$$

As a result, one obtains for the correlation function of the characteristic polynomials in the asymptotic limit $N \rightarrow \infty, x \gg 1$

$$
\left\langle 0\left|\bar{Z}^{n}(\theta) Z^{n}(0)\right| 0\right\rangle=N^{\frac{n^{2}}{\lambda}} \sum_{l=0}^{\infty}\left[F_{n}^{l}(\lambda)\right]^{2} \frac{e^{2 \pi i x l}}{(-2 \pi i x)^{\frac{(n-l)^{2}}{\lambda}}(2 \pi i x)^{\frac{l^{2}}{\lambda}}},
$$

where

$$
F_{n}^{l}(\lambda) \equiv\left(\begin{array}{c}
n \\
l
\end{array}\right) \frac{\prod_{a=1}^{l} \Gamma(1+a / \lambda) \prod_{a=1}^{n-l} \Gamma(1+a / \lambda)}{\prod_{j=a}^{n} \Gamma(1+a / \lambda)}=\left(\begin{array}{l}
n \\
l
\end{array}\right) \prod_{a=1}^{l} \frac{\Gamma(1+a / \lambda)}{\Gamma(1+(n-a+1) / \lambda)}
$$

and $F_{n}^{0}(\lambda)=1$. We have extended summation over $l$ to infinity in Eq. (25), since for an integer $n$ the binomial coefficient $\left(\begin{array}{l}n \\ l\end{array}\right)$ is identically zero for $l>n$. For RMT $(\lambda=1 / 2,1,2)$ Eq. (25) was derived recently from the saddle point treatment of the non-linear $\sigma$-model [9, 10]. In that case the factor $F_{n}^{l}(\lambda)$ is the volume of the degenerate coset space manifold $G(n) / G(l) G(n-l)$, where $G$ is an appropriate symmetry group, $G=S p, U, O$ for $\lambda=1 / 2.1 .2$ correspondingly.

Up to this moment all our calculations were performed for integer $n$ only. Next we shall continue analytically Eq. (25) to arbitrary real $n$ and then take the replica limit $n \rightarrow 0$. To this end we notice that

$$
\left(\begin{array}{l}
n \\
l
\end{array}\right) \equiv \frac{\Gamma(1+n)}{\Gamma(1+l) \Gamma(1+n-l)} \rightarrow n \frac{(-1)^{l+1}}{l}, \quad n \rightarrow 0
$$


This expression guarantees cancellation of the factor $n^{-2}$ in Eq. (14) for small $n$. As a result one obtains for $l \geq 1$ and $n \rightarrow 0$

$$
F_{n}^{l}(\lambda)=n \frac{(-1)^{l+1}}{l} \prod_{a=1}^{l} \frac{\Gamma(1+a / \lambda)}{\Gamma(1-(a-1) / \lambda)}+O\left(n^{2}\right) .
$$

Finally, employing Eq. (14) and taking the replica limit, one finds for the correlation function

$$
R(x)=-\frac{1}{2 \pi^{2} \lambda x^{2}}+2 \sum_{l=1}^{\infty} \frac{d_{l}^{2}(\lambda)}{(2 \pi x)^{2 l^{2} / \lambda}} \cos (2 \pi l x),
$$

where

$$
d_{l}(\lambda)=\frac{\prod_{a=1}^{l} \Gamma(1+a / \lambda)}{\prod_{a=1}^{l-1} \Gamma(1-a / \lambda)}=\Gamma\left(1+\frac{l}{\lambda}\right) \prod_{a=1}^{l-1}\left(\frac{a}{\pi \lambda}\right) \sin \left(\frac{\pi a}{\lambda}\right) \Gamma^{2}\left(\frac{a}{\lambda}\right) .
$$

The first term on the r.h.s. of Eq. (29) comes from the $l=0$ contribution. It may be called replica symmetric, since all $n$ integrals are calculated around $k_{a}=0$ (the left "Fermi" point). The oscillatory contributions on the r.h.s. of Eq. (29) may be dubbed "replica non-symmetric", since in $l$ out of $n$ replica contribution to the integral comes from the vicinity of $k_{a}=1$ (the right "Fermi" point).

For rational coupling constant $\lambda=p / q$ the sine function in Eq. (30) vanishes for $a=p$. As a result, $d_{l}(p / q)=0$ for $l>p$ and therefore the series in Eq. (29) contain only $p$ oscillatory components. For this case our results Eqs. (29), (30) coincide exactly with the asymptotic expression for the density-density correlation function obtained by Ha [5]. In Ref. [5] the coefficients $d_{l}^{2}(\lambda)$

are given in terms of the Dotsenko-Fateev integrals. One must employ the integral identity of Forrester and Zuk [12 to show the equivalence of coefficients in Ref. [5] and Eq. (30). In fact our approach together with Ref. [5] may be considered as an independent evaluation of some of the Dotsenko-Fateev integrals. We believe that for irrational $\lambda$ the series in Eq. (29) is an asymptotic one, but its exact meaning and possible re-summation procedure requires more study.

\section{Discussion of the Results}

Let us briefly discuss the generalization of the above procedure to the dynamical correlation function $R(x, t)=\rho_{0}^{-2}\langle 0|\tilde{\rho}(x, t) \tilde{\rho}(0,0)| 0\rangle$. The characteristic polynomials and replica are introduced in exactly the same way as for the static correlation function. One should take into account that operators at different times do not commute and that $\rho_{-k}(t)=\rho_{k}^{\dagger}(t)$. As a result one finds (cf. Eqs. (14) and (16))

$$
R(x, t)=-\lim _{n \rightarrow 0} \frac{1}{2 \pi^{2} n^{2}} \frac{\partial^{2}}{\partial x^{2}} \sum_{(\kappa)}\left|\left\langle(\kappa)\left|Z^{n}(0)\right| 0\right\rangle\right|^{2} \cos \left(|\kappa| x / \rho_{0}\right) e^{-i t\left(E_{(\kappa)}-E_{0}\right)},
$$

where the energies of excited states, $E_{(\kappa)}$, are given in Appendix A. The matrix elements of $Z^{n}(0)$ and their thermodynamic limit are exactly the same as for the static case. The thermodynamic limit of the excitation energy in terms of hole momenta in the proper frame of reference is given by Eq. (46). As a result, the integral on the r.h.s. of Eq. (19) acquires the factor $\exp \left\{-2 \pi i\left[v_{s} t \sum_{a} k_{a}\left(1-k_{a}\right) \mp x \sum_{a} k_{a}\right]\right\}$, where $v_{s} \equiv 2 \pi \rho_{0}^{2} \lambda$ is the sound velocity in the rescaled coordinates.

Asymptotic calculation of the $n$-fold momenta integral in the limit $x \gg 1$ and/or $v_{s} t \gg$ 1 follows closely the static case. The only difference is that the product of Selberg integrals $2 I_{n-l}(-2 \pi i x) I_{l}(2 \pi i x)$ in Eq. (23) is substituted by $I_{n-l}\left(2 \pi i\left[v_{s} t-x\right]\right) I_{l}\left(2 \pi i\left[v_{s} t+x\right]\right)+(x \rightarrow-x)$. 
Performing the analytical continuation $n \rightarrow 0$ and employing Eq. (31), one finally finds for the dynamic correlation function

$$
R(x, t)=-\frac{1}{4 \pi^{2} \lambda}\left(\frac{1}{\left(x-v_{s} t\right)^{2}}+\frac{1}{\left(x+v_{s} t\right)^{2}}\right)+2 \sum_{l=1}^{\infty} \frac{d_{l}^{2}(\lambda)}{\left(4 \pi^{2}\left[x^{2}-v_{s}^{2} t^{2}\right]\right)^{l^{2} / \lambda}} \cos (2 \pi l x),
$$

where the coefficients $d_{l}(\lambda)$ are given by Eq. (30).

The method also enables one to calculate the single particle Green function of the model. As it was shown in Ref. [5] the annihilation operator of particle acting on the ground state of $N+1$ particles is proportional to the action of $Z^{\lambda}$ on the $N$ particle ground state, i.e.

$$
\left\langle 0\left|\Psi^{\dagger}(x, t) \Psi(0,0)\right| 0\right\rangle=(N+1) \frac{\mathcal{N}(\lambda, N+1)}{\mathcal{N}(\lambda, N)} \sum_{(\kappa)}\left|\left\langle(\kappa)\left|Z^{\lambda}(0)\right| 0\right\rangle\right|^{2} e^{i(2 \pi|\kappa| / N-\pi \lambda) x-i\left(E_{(\kappa)}-\mu\right) t},
$$

Therefore to evaluate the Green function one has to perform analytical continuation of (25) from integer $n$ to $n=\lambda$. This yields in the large $N$ limit

$$
\left\langle 0\left|\Psi^{\dagger}(x, t) \Psi(0,0)\right| 0\right\rangle=\frac{N}{2 \pi} \sum_{l=0}^{\infty}\left[F_{\lambda}^{l}(\lambda)\right]^{2}\left(\frac{1}{2 \pi i\left(v_{s} t-x\right)}\right)^{\frac{(l-\lambda)^{2}}{\lambda}}\left(\frac{1}{2 \pi i\left(v_{s} t+x\right)}\right)^{\frac{l^{2}}{\lambda}} e^{i 2 \pi(l-\lambda / 2) x+i \mu t}
$$

where the coefficients $F_{\lambda}^{l}(\lambda)$ are still given by $(26)$ with $n \rightarrow \lambda$. For rational $\lambda=p / q$ they vanish for $l>p$ as in the case of the density-density correlations, so the sum in (34) contains a finite number of terms.

In summary, the correlation functions of the integer powers $n$ of the spectral determinants may be evaluated as a sum over partitions with no more than $n$ columns. In the thermodynamic limit such a sum takes a form of the $n$-fold integral, which may be evaluated for the large inter-particle separation. The subsequent analytical continuation to non-integer $n$ results in the density-density correlation function $(n \rightarrow 0)$ or in the single particle Green function $(n \rightarrow \lambda)$. These calculations do not require rational values of the coupling constant $\lambda$ and are formally valid for any $\lambda$. The final expressions, however, take the form of divergent series for irrational $\lambda$, whose exact meaning is not clear at the moment. For rational $\lambda$ the results coincide with those of Ref. [5].

Discussions with B. Altshuler initiated this project. We are also indebted to Y. Avron, J. Feinberg, S. Fishman, B. Simons M. Zirnbauer and A. Ludwig for valuable conversations. This research was supported in part by the U.S.-Israel Binational Science Foundation (BSF), by the Minerva Center for Non-linear Physics of Complex Systems, by the Israel Science Foundation, by the Niedersachsen Ministry of Science (Germany) and by the Fund for Promotion of Research at the Technion.

\section{A Eigenstates of the Calogero-Sutherland model}

Consider action of the kinetic term of the Hamiltonian Eq. (11) on the ground state, Eq. (2), which is convenient to rewrite as $\exp \{\lambda \ln \Delta(z)\}$ :

$$
\sum_{j=1}^{N}\left(z_{j} \partial_{j}\right)^{2}[\Delta(z)]^{\lambda}=\sum_{j=1}^{N}\left[\lambda\left(z_{j} \partial_{j}\right)^{2} \ln \Delta(z)+\lambda^{2}\left(z_{j} \partial_{j} \ln \Delta(z)\right)^{2}\right][\Delta(z)]^{\lambda} .
$$

The logarithmic derivatives are evaluated as follows:

$$
\begin{aligned}
z_{j} \partial_{j} \ln \Delta(z) & =\sum_{i \neq j} \frac{z_{j}}{z_{j}-z_{i}} \\
\left(z_{j} \partial_{j}\right)^{2} \ln \Delta(z) & =\sum_{i \neq j} \frac{z_{j}}{z_{j}-z_{i}}-\sum_{i \neq j} \frac{z_{j}^{2}}{\left(z_{j}-z_{i}\right)^{2}} .
\end{aligned}
$$


As a result the action of kinetic term Eq. (35) is equivalent to the multiplication by the following factor

$$
\lambda(\lambda-1) \sum_{i \neq j} \frac{z_{i} z_{j}}{\left(z_{i}-z_{j}\right)^{2}}+\lambda^{2}\left(\sum_{i \neq j \neq k} \frac{z_{i}^{2}}{\left(z_{i}-z_{j}\right)\left(z_{i}-z_{k}\right)}+\frac{N(N-1)}{2}\right) .
$$

The first term in this expression cancels exactly the action of the interaction term of the Hamiltonian Eq. (1), while the second term may be easily shown to be a constant. That proves that the wave-function Eq. (2) is indeed an eigenstate of the Hamiltonian Eq. (1) with the energy

$$
E_{0}(\lambda)=\lambda^{2}\left(\frac{N(N-1)(N-2)}{3}+\frac{N(N-1)}{2}\right)=\frac{\lambda^{2} N(N-1)(2 N-1)}{6}
$$

which may be considered as a sum of $N$ independent one-particle energies $\epsilon_{j}=\lambda^{2}(N-j)^{2}$ for $j$ running from 1 to $N$. The normalization of the ground state wave function is obtained straightforwardly with the help of the Selberg integral [6]

$$
\int_{0}^{2 \pi} \frac{d^{N} \theta}{(2 \pi)^{N}} \prod_{j<i}\left|e^{i \theta_{i}}-e^{i \theta_{j}}\right|^{2 \lambda}=\frac{\Gamma(1+\lambda N)}{\Gamma^{N}(1+\lambda)}
$$

resulting in the expression (2) for the normalized ground state.

To construct the excited states of the CSM one multiplies the ground state wave function by some symmetric polynomials $\Psi(z) \sim J(z) \Psi_{0}(z)$. The action of the kinetic term on this wave function generates the term (37) through the action of derivatives on $\ln \Psi_{0}(z)$ and the term, which involves the derivatives of $\ln J(z)$, leading to the eigenvalue equation:

$$
\left(H_{0}+\lambda H_{1}\right) J(z)=\left(E-E_{0}\right) J(z),
$$

where

$$
H_{0}=\sum_{i}\left(z_{i} \partial_{i}\right)^{2}, \quad H_{1}=\sum_{i \neq j} \frac{z_{i}+z_{j}}{z_{i}-z_{j}}\left(z_{i} \partial_{i}-z_{j} \partial_{j}\right)
$$

Solutions to this differential equation $J(z)=J_{(\kappa)}^{1 / \lambda}(z)$ are the symmetric Jack polynomials 14] labeled by the quantum numbers $(\kappa)=\left(\kappa_{1}, \kappa_{2}, \ldots \kappa_{N}\right)$. These numbers are non-negative integers organized in the non-increasing sequence $\kappa_{1} \geq \kappa_{2} \geq \ldots \geq \kappa_{N} \geq 0$ are known as parts of the partition $(\kappa)$. Since Jack polynomials are homogeneous functions, under translations $z_{j}=e^{i \theta_{j}} \rightarrow$ $u z_{j}=e^{i\left(\theta+\theta_{j}\right)}$ they transform as

$$
J_{(\kappa)}^{1 / \lambda}(u z)=u^{|\kappa|} J_{(\kappa)}^{1 / \lambda}(z) .
$$

The total momentum of an excited state is therefore

$$
P_{(\kappa)}=P_{0}+|\kappa|,
$$

where $P_{0}=\lambda N(N-1) / 2$ is the total momentum of the ground state and $|\kappa|=\kappa_{1}+\kappa_{2}+\ldots+\kappa_{N}$. The energy of the excitation $(\kappa)$ is given by

$$
E_{(\kappa)}=E_{0}+\sum_{j=1}^{N}\left(\kappa_{j}^{2}+2 \lambda \kappa_{j}(N-j)\right) .
$$

Each term in this sum can be represented as an energy gain $\left(\lambda(N-j)+\kappa_{j}\right)^{2}-(\lambda(N-j))^{2}$ of particle excited by momentum $\kappa_{j}$ from its ground state momentum $\lambda(N-j)$.

It is customary to represent partitions $(\kappa)$ by Young diagrams, a graph with $\kappa_{j}$ cells in the $j$-th row. A dual partition is obtained by using the numbers of cells, $\tilde{\kappa}_{a}$, in the $a$-th column of the Young diagram. 


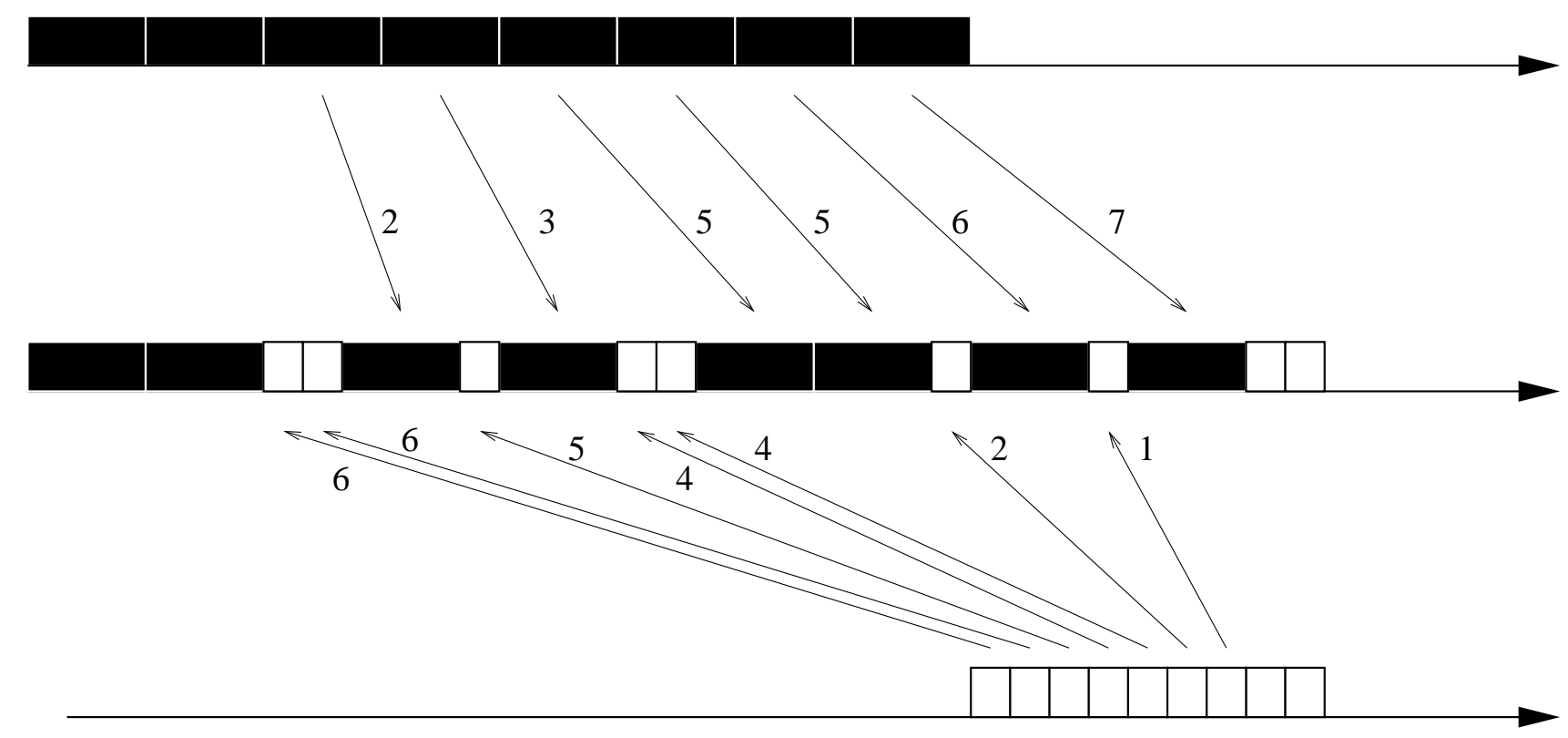

Figure 2: Another graphical representation of an excited state depicted in the previous figure. The excitations of particles and holes from their ground state position are related through the duality of the partitions. The number of particles is $N=8$ and the coupling constant $\lambda=3$.

For example, the partition dual to $(\kappa)=(7,6,5,5,3,2)$ ( the one depicted in Fig. 1 ) is $(\tilde{\kappa})=(6,6,5,4,4,2,1)$. It can be represented by transposing the Young diagram for $(\kappa)$. The dual partition can be given a nice interpretation in terms of hole excitations. Consider another graphical representation of the eigenstates of the Calogero-Sutherland model shown in Fig. 2 . Each particle in the ground state is represented by black box of length $\lambda$, which corresponds to the momentum $\lambda(N-j)$ added to the ground state. The excited state described by partition $(\kappa)$ is represented by moving $j$-th particle $\left(j=1\right.$ corresponds to the uppermost particle) by $\kappa_{j}$ units up. Some holes of integer length will appear between the particles. Recording the shifts of the holes from their ground state positions, one finds that they are described by the partition $(\tilde{\kappa})$ dual to $(\kappa)$ as depicted in Fig. 2. In terms of the dual partition $(\tilde{\kappa})$ the energy (44) of the excitation reads

$$
E_{(\kappa)}-E_{0}=\sum_{a}\left(2 \lambda \tilde{\kappa}_{a}(N+j / \lambda-(1+1 / \lambda) / 2)-\lambda \tilde{\kappa}_{a}^{2}\right),
$$

while the total momentum is given by $P_{(\kappa)}-P_{0}=|\tilde{\kappa}|$, since the number of cells can be counted row-wise as well as column-wise. In the limit of large $N$ the leading behavior of Eq. (45) is obtained by rescaling $\tilde{\kappa}_{a}=N k_{a}$ and reads $E_{(\kappa)}-E_{0} \rightarrow N^{2} \sum_{a} \lambda k_{a}\left(2-k_{a}\right)$. Here we must recall that we have chosen to work with the ground state with non-zero total momentum. That is equivalent to describing a system with zero-momentum ground state from the moving frame. Translation back to the frame where the ground state has zero momentum changes (only) expression for the excitation energy to

$$
E_{(\kappa)}-E_{0} \rightarrow N^{2} \sum_{a} \lambda k_{a}\left(1-k_{a}\right)
$$

This is the expression we use to calculate the dynamical correlation function, which belongs therefore to the rest frame of the ground state. 
The correspondence between dual partition and hole excitations is a non trivial fact and is crucial in our approach to the Calogero-Sutherland model.

\section{B Matrix Elements}

Here we describe how various scalar products between symmetric functions can be represented in terms of partitions. Let us denote by $j=1, \ldots, N$ the vertical coordinate and by $a=1,2, \ldots$ the horizontal coordinate of the cell in a Young diagram. The cell $(1,1)$ is the upper left corner of the partition. Then the symbols \{\}$_{(\kappa)}^{\lambda}$ and []$_{(\kappa)}^{\lambda}$ are defined by the following product over all cells in the Young diagram for $(\kappa)$ :

$$
\begin{aligned}
\{s\}_{(\kappa)}^{\lambda} & \equiv \prod_{j, a \in(\kappa)}(s-\lambda(j-1)+(a-1)) \\
{[s]_{(\kappa)}^{\lambda} } & \equiv \prod_{j, a \in(\kappa)}(s+(a-1) / \lambda-(j-1)) .
\end{aligned}
$$

The Jack polynomials are orthogonal with respect to the scalar product

$$
\int_{0}^{2 \pi} \frac{d^{N} \theta}{(2 \pi)^{N}} \prod_{j<l}\left|e^{i \theta_{l}}-e^{i \theta_{j}}\right|^{2 \lambda} J_{(\kappa)}^{1 / \lambda}(\bar{z}) J_{\left(\kappa^{\prime}\right)}^{1 / \lambda}(z)=\frac{j_{(\kappa)}^{\lambda}[N]_{(\kappa)}^{\lambda}}{[N+1 / \lambda-1]_{(\kappa)}^{\lambda}} \delta_{(\kappa),\left(\kappa^{\prime}\right)},
$$

where the factor $j_{(\kappa)}^{\lambda}$ may be written as the product over the cells of the Young diagram:

$$
j_{(\kappa)}^{\lambda}=\prod_{j, a \in(\kappa)}\left(\tilde{\kappa}_{a}-j+\left(\kappa_{j}-a\right) / \lambda+1 / \lambda\right)\left(\tilde{\kappa}_{a}-j+\left(\kappa_{j}-a\right) / \lambda+1\right) .
$$

Employing these expressions, one finds for the normalization constant $\mathcal{N}_{(\kappa)}$ of excited states of the CSM (cf. Eq. (5)):

$$
\mathcal{N}_{(\kappa)}=\frac{[N+1 / \lambda-1]_{(\kappa)}^{\lambda}}{j_{(\kappa)}^{\lambda}[N]_{(\kappa)}^{\lambda}}
$$

Jack polynomials form an orthogonal basis in the vector space of symmetric functions and can be used to expand any symmetric function. For example, the power sums, representing the Fourier coefficients of the density operator, Eq. (8), are given by the following linear combination of Jack polynomials:

$$
\sum_{j} z_{j}^{k}=\frac{k}{\lambda} \sum_{|\kappa|=k} \frac{\left[0^{\prime}\right]_{(\kappa)}^{\lambda}}{j_{(\kappa)}^{\lambda}} J_{(\kappa)}^{1 / \lambda}(z) .
$$

The prime in $\left[0^{\prime}\right]_{(\kappa)}^{\lambda}$ denotes that the cell $(1,1)$ is not included in the product in Eq. (48). More important for our discussion is the expansion of any power of the characteristic polynomials:

$$
Z^{s}(0)=\prod_{j}\left(1-z_{j}\right)^{s}=\sum_{(\kappa)} \frac{\{-s\}_{(\kappa)}^{\lambda}}{\lambda^{|\kappa|} j_{(\kappa)}^{\lambda}} J_{(\kappa)}^{1 / \lambda}(z) .
$$

In terms of normalized wave functions of the CSM, Eq. (5), the action of the $s$-power of the characteristic polynomial operator on the ground state may be written as the following linear combination:

$$
Z^{s}(\theta)|0\rangle=\sum_{(\kappa)} A_{(\kappa)}(\lambda, N)\{-s\}_{(\kappa)}^{\lambda} e^{-i|\kappa| \theta}|(\kappa)\rangle,
$$

where

$$
A_{(\kappa)}(\lambda, N)=\lambda^{-|\kappa|}\left(\frac{[N]_{(\kappa)}^{\lambda}}{j_{(\kappa)}^{\lambda}[N+1 / \lambda-1]_{(\kappa)}^{\lambda}}\right)^{1 / 2}
$$




\section{The large $N$ limit}

In this appendix we evaluate the thermodynamic limit $N \rightarrow \infty$ for the correlation function of the characteristic polynomials, Eq. (17). To this end we need to consider the partition-dependent coefficient

$$
\frac{\lambda^{-2|\kappa|}}{j_{(\kappa)}^{\lambda}} \frac{[N]_{(\kappa)}^{\lambda}}{[N+1 / \lambda-1]_{(\kappa)}^{\lambda}}\left[\{-n\}_{(\kappa)}^{\lambda}\right]^{2} .
$$

Let us start with $\{-n\}_{(\kappa)}^{\lambda}$ given by the product (47) over cells of the Young diagram representing $(\kappa)$. It is clear from this definition that $\{-n\}_{(\kappa)}^{\lambda}=0$ if $\kappa_{1}>n$. One can choose the heights $0 \leq \tilde{\kappa}_{a} \leq N ; a=1, \ldots, n$ of the first $n$ columns as the coordinates, since only the partitions with at most $n$ columns contribute. In these coordinates one can rewrite

$$
\{-n\}_{(\kappa)}^{\lambda}=(-\lambda)^{|\kappa|} \prod_{a=1}^{n} \prod_{j=1}^{\tilde{\kappa}_{a}}((n-a+1) / \lambda+j-1)=(-\lambda)^{|\kappa|} \prod_{a=1}^{n}((n-a+1) / \lambda)_{\tilde{\kappa}_{a}} .
$$

Here we have used the definition of the Pochhammer's symbol:

$$
(z)_{n} \equiv z(z+1)(z+2) \ldots(z+n-1)=\frac{\Gamma(z+n)}{\Gamma(z)} ; \quad(z)_{0}=1 .
$$

As a result, one finds

$$
\left[\{-n\}_{(\kappa)}^{\lambda}\right]^{2}=\lambda^{2|\kappa|} \prod_{a=1}^{n} \frac{\Gamma^{2}\left(\tilde{\kappa}_{a}+(n-a+1) / \lambda\right)}{\Gamma^{2}((n-a+1) / \lambda)} .
$$

The factors $[N]_{(\kappa)}^{\lambda}$ and $[N+1 / \lambda-1]_{(\kappa)}^{\lambda}$ are calculated in the same way by writing the products, Eq. (48), column-wise:

$$
\begin{aligned}
{[N]_{(\kappa)}^{\lambda}=\prod_{a=1}^{n} \prod_{j=1}^{\tilde{\kappa}_{a}}(N+(a-1) / \lambda-(j-1)) } & =\prod_{a=1}^{n} \frac{\Gamma(N+a / \lambda+1-1 / \lambda)}{\Gamma\left(N-\tilde{\kappa}_{a}+a / \lambda+1-1 / \lambda\right)} ; \\
{[N+1 / \lambda-1]_{(\kappa)}^{\lambda}=\prod_{a=1}^{n} \prod_{j=1}^{\tilde{\kappa}_{a}}(N+a / \lambda-j) } & =\prod_{a=1}^{n} \frac{\Gamma(N+a / \lambda)}{\Gamma\left(N-\tilde{\kappa}_{a}+a / \lambda\right)} .
\end{aligned}
$$

Next we calculate the factor $j_{(\kappa)}^{\lambda}$ defined in Eq. (50). We divide the Young diagram representing the partition $(\kappa)$ into $n$ rectangles having the fixed width $b=1,2, \ldots, n$ and height $\tilde{\kappa}_{b}-\tilde{\kappa}_{b+1}$ as shown in Fig. 3. Note that when $\tilde{\kappa}_{b}=\tilde{\kappa}_{b+1}$ the rectangle $b$ has zero height. With these notations the expression (50) can be written as a product over the rectangles:

$$
\begin{aligned}
j_{(\kappa)}^{\lambda} & =\prod_{b=1}^{n} \prod_{a=1}^{b} \prod_{j=\tilde{\kappa}_{b+1}+1}^{\tilde{\kappa}_{b}}\left(\tilde{\kappa}_{a}-j+(b-a+1) / \lambda\right)\left(\tilde{\kappa}_{a}-j+1+(b-a) / \lambda\right) \\
& =\prod_{b=1}^{n} \prod_{a=1}^{b} \frac{\Gamma\left(\tilde{\kappa}_{a}-\tilde{\kappa}_{b+1}+(b-a+1) / \lambda\right) \Gamma\left(\tilde{\kappa}_{a}-\tilde{\kappa}_{b+1}+1+(b-a) / \lambda\right)}{\Gamma\left(\tilde{\kappa}_{a}-\tilde{\kappa}_{b}+(b-a+1) / \lambda\right) \Gamma\left(\tilde{\kappa}_{a}-\tilde{\kappa}_{b}+1+(b-a) / \lambda\right)} .
\end{aligned}
$$

Renaming the running index $b+1 \rightarrow b$ and changing the order of the products, the numerator of the last expression may be written as

$$
\begin{aligned}
& \prod_{a=1}^{n} \Gamma\left(\tilde{\kappa}_{a}-\tilde{\kappa}_{n+1}+(n+1-a) / \lambda\right) \Gamma\left(\tilde{\kappa}_{a}-\tilde{\kappa}_{n+1}+1+(n-a) / \lambda\right) \\
\times & \prod_{b=a+1}^{n} \Gamma\left(\tilde{\kappa}_{a}-\tilde{\kappa}_{b}-(a-b) / \lambda\right) \Gamma\left(\tilde{\kappa}_{a}-\tilde{\kappa}_{b}-(a-b) / \lambda+1-1 / \lambda\right) .
\end{aligned}
$$




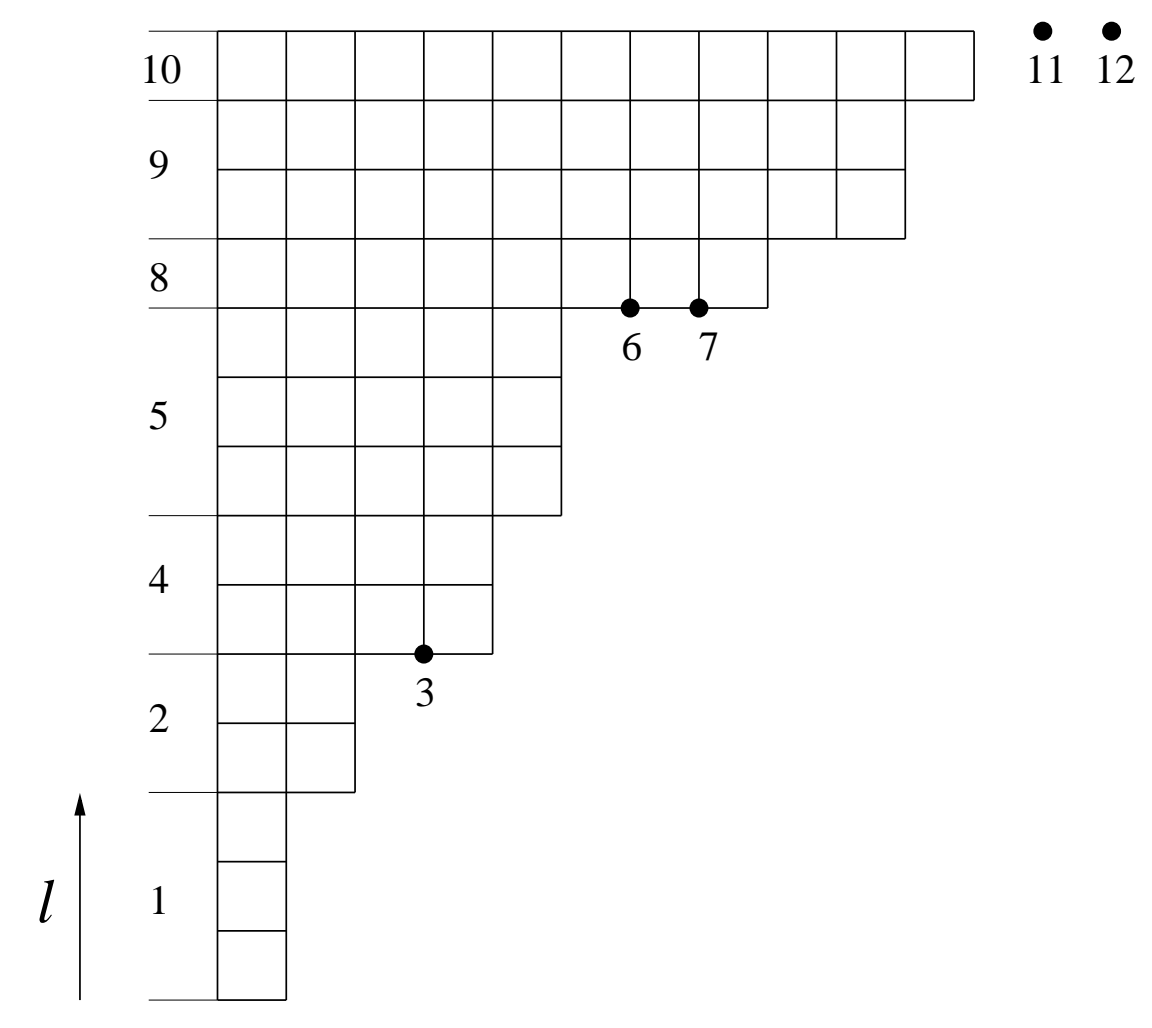

Figure 3: A Young diagram for $n=12$ divided in rectangles of fixed width $l$. Rectangles of zero height $l=3,6,7,11,12$ are represented by dots. 
In the denominator of Eq. (62) the order of the products is interchanged and the terms with $a=b$ are factored out, the result is

$$
\Gamma^{n}(1 / \lambda) \prod_{a=1}^{n} \prod_{b=a+1}^{n} \Gamma\left(\tilde{\kappa}_{a}-\tilde{\kappa}_{b}-(a-b) / \lambda+1 / \lambda\right) \Gamma\left(\tilde{\kappa}_{a}-\tilde{\kappa}_{b}-(a-b) / \lambda+1\right) .
$$

Finally, using the fact that $\tilde{\kappa}_{n+1}=0$, one obtains

$$
\begin{aligned}
j_{(\kappa)}^{\lambda} & =\frac{1}{\Gamma^{n}(1 / \lambda)} \prod_{a=1}^{n} \Gamma\left(\tilde{\kappa}_{a}+(n-a+1) / \lambda\right) \Gamma\left(\tilde{\kappa}_{a}+1+(n-a) / \lambda\right) \\
& \times \prod_{b=a+1}^{n} \frac{\Gamma\left(\tilde{\kappa}_{a}-\tilde{\kappa}_{b}-(a-b) / \lambda\right) \Gamma\left(\tilde{\kappa}_{a}-\tilde{\kappa}_{b}-(a-b) / \lambda+1-1 / \lambda\right)}{\Gamma\left(\tilde{\kappa}_{a}-\tilde{\kappa}_{b}-(a-b) / \lambda+1 / \lambda\right) \Gamma\left(\tilde{\kappa}_{a}-\tilde{\kappa}_{b}-(a-b) / \lambda+1\right)} .
\end{aligned}
$$

Combining the above expressions together one finally obtains for the coefficient Eq. (56):

$$
\begin{aligned}
& \prod_{a=1}^{n} \quad \frac{\Gamma(1 / \lambda)}{\Gamma^{2}((n-a+1) / \lambda)} \\
& \times \prod_{a=1}^{n} \quad \frac{\Gamma\left(\tilde{\kappa}_{a}+(n-a) / \lambda+1 / \lambda\right)}{\Gamma\left(\tilde{\kappa}_{a}+(n-a) / \lambda+1\right)} \frac{\Gamma\left(N-\tilde{\kappa}_{a}+a / \lambda\right)}{\Gamma\left(N-\tilde{\kappa}_{a}+a / \lambda+1-1 / \lambda\right)} \frac{\Gamma(N+a / \lambda+1-1 / \lambda)}{\Gamma(N+a / \lambda)} \\
& \times \prod_{b=a+1}^{n} \quad \frac{\Gamma\left(\tilde{\kappa}_{a}-\tilde{\kappa}_{b}-(a-b) / \lambda+1 / \lambda\right) \Gamma\left(\tilde{\kappa}_{a}-\tilde{\kappa}_{b}-(a-b) / \lambda+1\right)}{\Gamma\left(\tilde{\kappa}_{a}-\tilde{\kappa}_{b}-(a-b) / \lambda\right) \Gamma\left(\tilde{\kappa}_{a}-\tilde{\kappa}_{b}-(a-b) / \lambda+1-1 / \lambda\right)} .
\end{aligned}
$$

The thermodynamic limit, $N \rightarrow \infty$, is obtained with the help of the following asymptotic relation:

$$
\lim _{|z| \rightarrow \infty} \frac{\Gamma(z+a)}{\Gamma(z)}=z^{a} .
$$

Rescaling the column heights as $\tilde{\kappa}_{a}=N k_{a}$, one obtains for the thermodynamic limit of the expression (66)

$$
\frac{N^{\frac{n^{2}}{\lambda}}}{N^{n}} \prod_{a=1}^{n} \frac{\Gamma(1 / \lambda)}{\Gamma^{2}((n-a+1) / \lambda)} \prod_{1 \leq a<b \leq n}\left(k_{a}-k_{b}\right)^{2 / \lambda} \prod_{a=1}^{n} k_{a}^{1 / \lambda-1}\left(1-k_{a}\right)^{1 / \lambda-1} .
$$

We relax the requirement $k_{1} \geq k_{2} \geq \ldots \geq k_{n}$ allowing all the variables to run independently between 0 and 1 . To do so we have to divide by $n$ ! and take the absolute value of the Vandermonde determinant:

$$
\prod_{1 \leq a<b \leq n}\left(k_{a}-k_{b}\right)^{2 / \lambda} \rightarrow\left|\Delta_{n}(k)\right|^{2 / \lambda} .
$$

Finally, changing the sum over partitions to the integrals over $k_{a}$ and absorbing the factor $N^{-n}$ into the integration measure, one recovers Eqs. (19) and (20).

\section{References}

[1] F. Calogero, J.Math.Phys 10, 2191 (1969), B. Sutherland, Phys. Rev. A 4, 2019 (1971).

[2] P. J. Forrester, Nucl.Phys. B 388, 671, (1992), P.J. Forrester, Phys.Lett. A 179, 127 (1993).

[3] B. D. Simons, P. A. Lee and B. L. Altshuler, Phys.Rev.Lett. 70, 4122 (1993). 
[4] F. D. M. Haldane, in the Proceedings of the 16th Taniguchi Symposium, Kashikijima, Japan, October 29-29, 1993, editors A. Okiji and N. Kawakami, Springer-Verlang, 1994.

[5] Z. N. C. Ha, Phys.Rev.Lett. 73, 1574 (1994), Z. N. C. Ha, Quantum Many-Body Systems in One Dimension, World Scientific, Singapore, 1996.

[6] M. L. Mehta, Random Matrices, second edition, Academic Press, Boston, 1991.

[7] S. F. Edwards and P. W. Anderson, J. Phys. F 5, 89 (1975).

[8] J. J. M. Verbaarschot and M. R. Zirnbauer, J. Phys. A 17, 1093 (1985).

[9] A. Kamenev and M. Mezard, J.Phys. A 32, 4373 (1999), A. Kamenev and M. Mezard, Phys.Rev. B 60, 3944 (1999).

[10] I. V. Yurkevich and I. V. Lerner, Phys.Rev. B 60, 3955 (1999).

[11] M.Zirnbauer, cond-mat/9903338

[12] P. J. Forrester and J.A. Zuk, Nucl.Phys. B 473, 616 (1996).

[13] H. Jack, Proc. Roy. Soc. Edinburgh Sect. A 691 (1969-1970), R. P. Stanley, Adv. Math. 77, 76 (1989).

[14] I. G. Macdonald, Symmetric functions and Hall polynomials, Oxford Univ. Press, Oxford, 1979.

[15] This ground state breaks the symmetry $\lambda \rightarrow 1-\lambda$ of the Hamiltonian (10).

[16] G. E. Andrews, The Theory of Partitions, Addison-Wesley, Reading, 1976.

[17] M. Zirnbauer, private communication.

[18] J. P. Keating and N. C. Snaith, Comm.Math.Phys. 214, 57 (2000).

[19] P. J.Hanlon, R. P. Stanley, and J. R. Stembridge, Contemp.Math. 138, 151 (1992).

[20] Z. Yan, Contemp.Math. 138, 239 (1992).

[21] The fermionic replica corresponds to the positive integer power $n$ of the spectral determinant, while the bosonic replica - to the negative one. The difference is crucial since as was shown in [8] the bosonic replica solution do not contain the oscillatory terms which appear due to the finite $N$. The negative power $n$ does not restrict the number of holes in the excited states and the analysis based on the hole momenta is not possible. 